\title{
Editorials
}

\section{The future of primary care?}

\section{Reflections on the Primary Care Workforce Commission report}

There is a general consensus that there is a crisis in general practice in the UK with a 'perfect storm' of workload pressures, inadequate funding, and low morale. Responding to a catastrophic fall in recruitment and poor retention of experienced GPs, a national 10-point 'general practice workforce action plan' has been laid out by NHS England (NHSE). Health Education England commissioned Professor Martin Roland to chair a Primary Care Workforce Commission to gather evidence and make recommendations not only to address the current crisis, but also to project forward and attempt to 'future proof' the profession. Proposed changes are intended to support the development of 'a modern primary healthcare system' in the context of "challenging and fulfilling careers for health professionals', and maintaining pride in the 'standard of care' delivered to patients. ${ }^{2}$

Professor Roland has the right credentials for the job, having maintained a clinical workload as a GP alongside his distinguished academic career. Furthermore, during his leadership, the National Primary Care Research and Development Centre undertook a portfolio of studies addressing primary care workforce issues, including skill mix in primary care, equity in the geographical distribution of GPs, and GP satisfaction and work stress. . $^{3-6}$ In this Commission, his team of equally experienced colleagues, academic GPs, and external experts took evidence from a wide range of bodies, including individual GP practices, clinical commissioning groups (CCGs), national bodies such as the Family Doctor Association and the Royal College of Nursing, and charities such as National Voices, and made site visits to observe innovative practices and organisations.

The focus of these recommendations is on 'primary care' rather than general practice, but, while the report rejects uniformity in favour of locally appropriate variations, it identifies GP practices as vital units in the coordination and delivery of safe and effective primary health care. The report emphasises a multidisciplinary team approach based around general practices, with GPs, nurses, pharmacists, physiotherapists, and new types of professionals, such as physician associates (PAs), working across organisational boundaries to provide personalised care.

\section{"There is a general consensus that there is a crisis in general practice in the UK with a perfect storm of workload pressures, inadequate funding, and low morale.}

The report commends innovative practices already introduced piecemeal across the UK, and suggests that, rather than radical redesign, what is needed is spread of existing successful models.

In assessing the extensive recommendations, summarised in Boxes 1 and 2, it is perhaps useful to think of them from three viewpoints: organisational implications for practices; the perspective of GPs and other practice and community staff; and the perspective of patients.

\section{ORGANISATIONAL IMPLICATIONS}

In this vision, practices with a more diverse workforce will provide care in ways that chime with practice concerns and priorities. For example, pre-appointment triage will enable the provision of longer appointments that we know can facilitate patient-centred care for patients with complex problems.? Similarly, many practices and CCGs already benefit from working closely with local community pharmacists. ${ }^{8}$ Protected time for staff to evaluate their care and think proactively about implementing improvements is seen as important, with the Commission arguing that this may help alleviate pressures. However, it is difficult to see how hard-pressed staff can take time out to accomplish this without more investment and external support. CCGs are currently taking over responsibility for commissioning primary care services, which may allow for additional resources and shared access to training. However, CCGs are themselves stretched by new responsibilities and limited resources, which

\section{Box 1. Recommendations 1: strategies to restructure care delivery in general practices}

\section{Multidisciplinary workforce}

- Increase recruitment and retention of GPs via NHS England's 10-point plan

- Similar measures for practice nurses

- Greater involvement of pharmacists in general practices, with strengthened collaboration between practices and community pharmacies

- Greater use of skill mix, bringing in physician associates and healthcare assistants, and evaluating a potential role for paramedics in acute home visits

- New staff support roles to remove the 'admin burden' from GPs

- Evaluation and testing of the greater use of direct referral to allied health professionals such as physiotherapists and occupational therapists

- 24-hour community nursing services to support out-of-hospital care

- Research to assess the relative costs versus benefits of skilled versus unskilled triage of requests for out-of-hours care

- General practices encouraged to take a more analytical approach to the needs of their population to ensure that care meets those needs

- Protected time for practices to develop their teamwork and quality improvement activities

\section{Quality and safety in primary care}

- A significant proportion of face-to-face consultations should be longer than 10 minutes

- Monitoring of access targets should focus on the ability of patients to book an appointment with the doctor or nurse of their choice to facilitate continuity of care

- Quality data about general practice care should be publicly available

\section{Better use of technology}

- E-mail contact between GPs and hospital specialists should become routine

- Use of a common record between primary and community nursing staff

- Single point of access to out-of-hours care 


\section{Box 2. Recommendations 2: generating capacity, building connections, and reducing inequalities}

\section{Education and training}

- Structured training opportunities should be available for all primary care staff, including leadership training for those leading federations

- Pre- and post-registration placements in primary care should be routine for pharmacists and nurses

- Pharmacy students should all have access to pre-registration experience in community pharmacies

- There should be joint training opportunities for health and social care staff, with establishment of community education provider networks' to coordinate this

\section{Integrating care}

- Greater contact between primary care staff and specialists, with specialists working in new ways to support primary care teams

- Those contracted to provide out-of-hours care should be required to demonstrate their ability to link with other primary care providers, with an expectation that this will encourage new providers (such as federations of multispecialty community providers) to provide out-of-hours care

- Single point of access for community and social care services for urgent assessment, with co-location of relevant staff

\section{Federations and networks of practices}

- The development of networks of practices should be encouraged

- Federations should actively seek out links with patient and community groups

\section{Population groups with particular needs}

- Inequalities in the distribution of healthcare professionals should be actively addressed

- GP practices should have access to a named consultant psychiatrist and a named mental health worker

- GP practices should have access to a named paediatrician and a named children's nurse

- Clinical commissioning groups should address the needs of vulnerable groups, such as the homeless and substance misusers

- Care for patients in care homes should be the responsibility of one general practice and one community pharmacy, with GPs within the practice taking lead responsibility

- Patients at the end of their lives should have a named lead care worker

- Teams caring for patients at the end of their lives should have 24-hour access to care plans, night sitting services, and community nurses

\section{Better data}

- Better routine data about workforce and their work are urgently needed

- New service models should have evaluation established from the beginning

militates against their contribution in the absence of significant investment. Although direct communication with specialist consultants by e-mail and telephone should be the norm, other recommendations seem a little more problematic. It is not clear from the evidence that relocating specialists into primary care is time or cost efficient, 3,6 while the evidence relating to the use of PAs in the UK is thin at best, ${ }^{10}$ and would benefit from further evaluation.

\section{IMPLICATIONS FOR GPS}

From the point of view of GPs, these recommendations suggest a future role that is somewhat different from that which they play now. The report implies the development of a GP consultant role, with senior GPs taking a more coordinating and consultative approach, overseeing a team of healthcare providers including nurses, pharmacists, and PAs. Although some GPs may embrace this new role, others may regret the loss of their place as the first port of call where skill, insight, and expertise can be vital; most GPs can recall examples of apparently simple and routine consultations that uncovered something more serious. Nuanced histories and examination cannot be reliably achieved through triage, with the result that pre-consultation segregation by presenting problem may put comprehensive and skilled assessment at risk, as well as limiting patients' choice of available healthcare provider and continuity of care. ${ }^{11,12}$ This model leads to further questions about responsibility; the report is silent on the question of who bears ultimate responsibility for independent work delivered by the extended primary care team. On the wider objective of providing 'challenging and satisfying careers' it remains unclear how well prepared the existing or future GP workforce will be for a role that concentrates on clinical complexity and supervisory duties, while removing cases that can be readily diagnosed and resolved. Individual preferences and abilities may thrive at different levels within practices; however, it seems likely that the introduction of structured progression for GPs will be necessary to support development of confidence and expertise alongside acceptance of new definitions of being a GP. The old model of additional payments for length of service has clearly outlived its usefulness, but it is not yet clear how a more differentiated GP workforce could function or be rewarded.

\section{IMPLICATIONS FOR PATIENTS}

Patients are broadly familiar with existing appointment systems, which, in many practices, already include alternatives to GPs. Implementation of these recommendations would, however, increasingly limit their appointment choices by diverting acute problems to a PA or nurse practitioner. Further restrictions may result if requests for home visits are routinely transferred for paramedic assessment. Innovative technological solutions, including e-mail exchanges with patients, Skype consultations, and online booking, will be welcomed by many, but technology can exclude, whether through choice, communication difficulties, or other obstacles. Because unintended consequences can result from such changes, careful assessment in advance of change will be necessary to avoid improving some types of access at the expense of others. For example, increasing availability of short-notice appointments using the 'Advanced Access' model improved immediate access at the expense of the ability to book a convenient appointment in advance. ${ }^{13}$

The report contains some low-cost and easy-to-implement changes: direct e-mail contact between GPs and specialists should be routine, and enhanced recruitment of community nurses through early exposure to general practice should build on existing training expertise in general practices. Other apparently simple innovations may prove more difficult to implement. It seems obvious that practices, community nurses, and out-of-hours providers should have shared access to common medical records, but current systems would require significant modification to allow full interoperability, and changing to a single common system would be expensive 


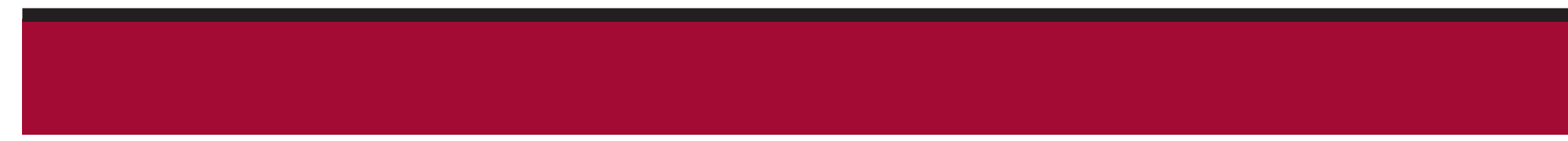

"The innovations and changes discussed here are all feasible, given investment. Without it, it is hard to see how primary care as we know it can be sustained."

and difficult to implement. Enhanced interoperability may be more manageable than uniformity, with incentives and penalties to encourage manufacturers to share technology and expertise. However, research has shown that concerns about information governance and about appropriate use of systems can make shared record use difficult to achieve, even where systems are compatible. ${ }^{14}$ The apparently simple idea that GP time will be released by administrative staff trained to take responsibility for tasks, such as responding to blood test results or hospital letters, may also turn out to be problematic, because a significant proportion of these require background clinical knowledge to ensure patient safety. Stronger evidence is necessary to guide safe and effective implementation.

In spite of these caveats, the Commission report represents a comprehensive and brave attempt to sketch out a sustainable future for primary care that builds on established GP practice units and locally developed networks. It is to be welcomed as an important contribution to solving the current crisis. The report starts with a diagnosis of 'the problem', highlighting

\section{ADDRESS FOR CORRESPONDENCE}

\section{Kath Checkland}

University of Manchester, Centre for Primary

Care, 5th Floor Williamson Building, Oxford Road,

Manchester, M13 9PL, UK.

\section{E-mail: kath.checklandamanchester.ac.uk}

historical lack of investment in general practice and community services as the number one issue. The innovations and changes discussed here are all feasible, given investment. Without it, it is hard to see how primary care as we know it can be sustained.

\section{Kath Checkland,}

Professor of Health Policy and Primary Care, Centre for Primary Care, Institute of Population Health, University of Manchester, Manchester.

\section{Sharon Spooner,}

NIHR Academic Clinical Lecturer, Centre for Primary Care, Institute of Population Health, University of Manchester, Manchester.

\section{Provenance}

Commissioned; not externally peer reviewed.

DOI: 10.3399/bjgp15X686773

\section{REFERENCES}

1. NHS England. Building the workforce - the new deal for general practice. http://www. england.nhs.uk/commissioning/wp-content/ uploads/sites/12/2015/01/building-the-

workforce-new-deal-gp.pdf laccessed 26 Aug 2015).

2. Health Education England. The future of primary care: creating teams for tomorrow. http://hee.nhs.uk/wp-content/blogs.dir/321/ files/2015/07/The-future-of-primary-care.pdf laccessed 25 Aug 2015).

3. Black M, Leese B, Gosden T, Mead N. Specialist outreach clinics in general practice: what do they offer? Br J Gen Pract 1997; 47(422): 558-561

4. Hann M, Gravelle $H$. The maldistribution of general practitioners in England and Wales: 1974-2003. Br J Gen Pract 2004; 54(509): 894-898.

5. Sibbald B, Bojke C, Gravelle H. National survey of job satisfaction and retirement intentions among general practitioners in England. BMJ 2003; 326(7379): 22.

6. Sibbald B, Pickard S, McLeod H, et al. Moving specialist care into the community: an initial evaluation. J Health Serv Res Policy 2008; 13(4): 233-239.

7. Wilson A, Childs S. The relationship between consultation length, process and outcomes in general practice: a systematic review. $\mathrm{Br} J \mathrm{Gen}$ Pract 2002; 52(485): 1012-1020.

8. Lai K, Howes K, Butterworth C, Salter M. Lewisham Integrated Medicines Optimisation Service: delivering a system-wide coordinated care model to support patients in the management of medicines to retain independence in their own home. Eur J Hosp Pharm 2015; 22(2): 98-101.

9. NHS England. Next steps towards primary care co-commissioning. 2014. http://wnw. england.nhs.uk/commissioning/wp-content/ uploads/sites/12/2014/11/nxt-steps-pccocomms.pdf laccessed 25 Aug 2015).

10. Drennan VM, Halter M, Joly L, et al. Physician associates and GPs in primary care: a comparison. Br J Gen Pract 2015; DOI: 10.3399/bjgp15X684877.

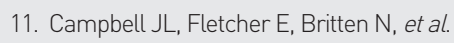
Telephone triage for management of sameday consultation requests in general practice (the ESTEEM trial): a cluster-randomised controlled trial and cost-consequence analysis. Lancet 2014; 384(9957): 1859-1868.

12. Murdoch J, Barnes R, Pooler J, et al. The impact of using computer decisionsupport software in primary care nurse-led telephone triage: interactional dilemmas and conversational consequences. Soc Sci Med 2015; 126: 36-47.

13. Salisbury C, Goodall S, Montgomery AA, et al. Does Advanced Access improve access to primary health care? Questionnaire survey of patients. Br J Gen Pract 2007; 57(541): 615-621.

14. CLAHRC-GM. NHS Greater Manchester primary care demonstrator evaluation. Final report. $2015 \mathrm{http}: / / c l a h r c-g m . n i h r . a c . u k / c m s /$ wp-content/uploads/PCDE-final-report-fullfinal.pdf (accessed 25 Aug 2015) 